\title{
Frontal EEG theta/beta ratio during mind wandering episodes
}

\author{
Dana van Son ${ }^{1,2}$, Frances M. De Blasio ${ }^{3}$, Jack S. Fogarty ${ }^{3}$, Angelos Angelidis ${ }^{1,2}$, Robert J. Barry ${ }^{3}$, \\ Peter Putman ${ }^{1,2}$ \\ ${ }^{1}$ Institute of Psychology, Leiden University, Leiden, The Netherlands \\ ${ }^{2}$ Leiden Institute for Brain and Cognition, Leiden, The Netherlands \\ ${ }^{3}$ Brain \& Behaviour Research Institute and School of Psychology, University of Wollongong, Wollongong, Australia
}

\begin{abstract}
Background: In resting-state EEG, the ratio between frontal power in the slow theta frequency band and the fast beta frequency band (the theta/beta ratio, TBR) has previously been negatively related to attentional control. Also, increased theta and reduced beta power were observed during mind wandering (MW) compared to episodes of focused attention. Thus, increased resting-state frontal TBR could be related to MW, suggesting that previously observed relationships between TBR and attentional control could reflect MW episodes increasing the average resting state TBR in people with low attentional control.

Goals: To replicate and extend the previous theta and beta MW effects for frontal TBR recordings and test if MW related changes in frontal TBR are related to attentional control.

Methods: Twenty-six healthy participants performed a 40-minute breath-counting task, after a baseline EEG recording, while EEG was measured and participants indicated MW episodes with button presses.

Results: Frontal TBR was significantly higher during MW episodes than during on-task periods. However, no relation between frontal TBR and attentional control was found.

Conclusions: This confirms that frontal TBR varies with MW episodes and that previous frontal TBRattentional control relations might be related to MW, though no direct evidence was found for this hypothesis.

199 words
\end{abstract}

Keywords: EEG theta/beta ratio, mind wandering, attentional control 
The electroencephalographic (EEG) signal represents the combined electrical fluctuations in membrane potentials generated from the interactions of the primary inhibitory and excitatory neurons (Gordon, 2000; Nunez, 1995) and can be decomposed into power estimates of different frequency bands. Typically measured under resting conditions, the ratio between the slow wave theta (4-7 Hz) and fast wave beta (13-30 Hz) band power, in other words the theta/beta ratio (TBR), has been utilized as a source of critical information about brain activity that may be associated with increased cognitive demand (Barry, Clarke, \& Johnstone, 2003). TBR has also been found to have a very high test-retest reliability (Angelidis, van der Does, Schakel, \& Putman, 2016; Keune, Hansen, Weber, Zapf, Habich, Muenssinger, Wolf, et al., 2017).

Several lines of evidence suggest that TBR is of interest when investigating attentional control. A robust finding, for example, is that TBR is increased in patients diagnosed with attentiondeficit/ hyperactivity disorder (ADHD; Arns, Conners, \& Kraemer, 2013; Barry et al., 2003). Additionally, TBR was negatively correlated with self-reported trait attentional control (using the Attentional Control Scale, or ACS; Derryberry \& Reed, 2002) in healthy participants (especially when controlling for an often-correlated measure of trait anxiety; Putman, van Peer, Maimari \& van der Werff, 2010; Putman, Verkuil, Arias-Garcia, Pantazi \& van Schie, 2014; Angelidis et al., 2016; van Son, Angelidis, Hagenaars, van der Does \& Putman, 2018a). Also, TBR was negatively related to objectively measured attentional control in multiple sclerosis patients with mild cognitive impairment (Keune et al., 2017). Furthermore, TBR was found to be positively correlated with a stress-induced decline in state attentional control (Putman et al., 2014). Frontal TBR has been suggested to reflect cortical-subcortical interactions associated with inhibitory functioning and cortical inhibition of subcortical processes (Knyazev, 2007; Schutter \& Knyazev, 2012; Putman et al., 2014). This could reflect voluntary top-down processes like attentional control carried out by the dorsolateral prefrontal cortex (Bishop, 2008; Gregoriou, Rossi, Ungerleider, \& Desimone, 2014) over automatic bottom-up processes mediated by limbic areas such as the anterior cingulate cortex and the amygdala, facilitating attention to salient information (Hermans, Henckens, Joëls \& Fernández, 2014).

Recent studies from our lab showed that TBR moderated attentional bias to stimuli of different threat levels (Angelidis, Hagenaars, van Son, van der Does \& Putman, 2018; van Son et al., 
2018a) as predicted for attentional control in influential models of attentional bias (Mogg \& Bradley, 1998, 2016). However, attentional bias does not solely include attentional processing of external stimuli. Anxious people, for example, also worry a lot, which represents biased internal activation of threatening cognitions in working memory, and shares mechanisms with biased attention (Hirsch \& Mathews, 2012). Worry can be seen as self-generated off-task thought, and is sometimes referred to as a 'negative form' of the umbrella term ‘mind wandering' (Ottaviani, Shahabi, Tarvainen, Cook, Abrams \& Shapiro, 2015).

Like worry, mind wandering (MW) episodes correspond to the emergence of task-unrelated affects and thoughts that draw attention away from the task at hand (Smallwood \& Schooler, 2006). MW can occur while performing a task, and is manifested as thinking of something else while executing a task (Mason, Norton, Van Horn, Wegner, Grafton, \& Macrae, 2007). It is also described as a deficit in working memory and attentional control (McVay \& Kane, 2009; Unsworth \& McMillan, 2014), and is a predictor of performance errors (Smallwood \& Schooler, 2006).

In a proof of principle study, Braboszcz and Delorme (2011) reported that higher EEG theta band power and lower EEG beta band power were related to a state of MW. Participants were asked to focus on counting their breaths and to press a button as soon as they became aware that their mind had wandered off task. EEG spectral analysis showed higher theta and lower beta (likely higher TBR) before the button press, but lower theta and higher beta (likely lower TBR) after the button press, when they again focused on breath counting. These results were observed for windows of a -8 to -2 second period before the button press and a 2 to 8 second period after the button press, omitting the four seconds surrounding the button press. These time-windows correspond with theoretical and empirical observations concerning short periods of low, but growing awareness and a shift in attentional orientation just before and after the button press respectively (Hasenkamp, WilsonMendenhall, Duncan \& Barsalou, 2012). The two seconds immediately before the button press were considered as 'participants becoming aware that their mind wandered off', and the two seconds immediately after the button press as 'getting back into breath-counting'.

As outlined above, MW itself is described as a deficit in working memory and attentional control (McVay \& Kane, 2009; Unsworth \& McMillan, 2014) and is a predictor for performance 
errors (Smallwood \& Schooler, 2006); TBRs relation to poor attentional control might therefore be associated with a higher tendency to mind wander during resting state, increasing the average TBR in people with low attentional control. Studying this hypothesis would greatly benefit our understanding of TBR in healthy people (e.g. Angelidis et al., 2016), people with mild cognitive impairments (Keune et al., 2017) and ADHD patients (Arns et al., 2013; Barry et al., 2003) amongst others. If the TBR-attentional control relationship reflects mainly changes in TBR when people engage in mind wandering episodes, it might also be interesting to consider possible interactions between the number of mind wandering episodes and the assumed TBR-attentional control correlation. Therefore, if the TBR-ACS relation is observed in our present sample (null-findings reported in Morillas-Romero, Tortella-Feliu, Bornas, \& Putman, 2015; Angelidis et al., 2018), the hypothesis regarding the underlying processes causing the relation between TBR and attentional control will be additionally explored.

The aim of the current study was to replicate and extend the design and results of Braboszcz and Delorme (2011) as pertaining to the MW related changes in EEG, in order to gain further insight into the role of TBR during MW episodes. Our primary hypotheses will be tested using frontal TBR, since previous studies examining TBR in relation to executive processes using healthy participants focused almost exclusively on frontal TBR (Putman et al., 2010, 2014; Angelidis et al., 2016, 2018; van Son et al., 2018a; Schutter \& van Honk, 2004; Schutter \& van Honk, 2005; Sari, Koster, Pourtois, \& Derakshan, 2016; Tortella-Feliu, Morillas-Romero, Balle, Llabrés, Bornas, \& Putman, 2014; Morillas-Romero et al., 2015). We hypothesized that:

I) Frontal TBR is higher during MW episodes than during on-task periods.

II) Baseline spontaneous frontal TBR is expected to negatively correlate with attentional control as measured by the ACS when controlling for trait anxiety.

III) The MW related changes in frontal TBR (assessed in hypothesis I) are related to baseline TBR during resting state and ACS.

IV) The MW related changes in frontal TBR (assessed in hypothesis I) mediate the correlation between baseline spontaneous TBR and ACS (hypothesis II).

The delta, theta, alpha and beta bands will also be exploratively investigated in the same design. It is 
yet to be verified if TBR at frontal regions is the optimal predictor of attentional control and MW, thus the present study additionally explores the topographical occurrence of MW-related TBR. Furthermore, after testing the hypotheses for the MW versus focused attention epochs corresponding to Braboszcz and Delorme’s (2011) analysis, we further explore effects of time within these 6 second epochs (pre- and post-button press) as visual inspection of the Braboszcz and Delorme (2011) data suggests that TBR increased following the button press. Finally, we correlated EEG data with the number of button presses that the participants made, as the occurrence of MW awareness might be related to the qualitative nature of mind wandering episodes; that is, less profound mind wandering might occur in participants who often become aware of their mind wandering.

\section{Methods}

\section{Participants}

Fifty-four female participants (between 18 and 30 years old) recruited at Leiden University took part in this study. Only females were included because of the low prevalence of men signing up to participate in the study. Exclusion criteria were factors which would likely adversely affect participation, EEG, or attention; these included severe physical or psychological dysfunction, and/or the use of psychotropic medication. As described in detail below, 28 participants were excluded because they retained too few $(<11)$ acceptable mind wandering epochs of acceptable EEG data quality. Informed consent was obtained prior to testing, and participants received a monetary reimbursement for their participation. The study was approved by the Leiden University local ethics review board.

Materials

\section{Questionnaires}

Participants completed the trait version of the State-Trait Anxiety Inventory (STAI-t; Spielberger, 1983) and the Attentional Control Scale (ACS; Derryberry \& Reed, 2002). The STAI-t assesses trait anxiety (20 items, range 20-80; Cronbach’s alpha in the current study $=0.88$ ), by 
indicating agreement with items like 'I feel nervous and restless' and 'I have disturbing thoughts' on a four-point Likert scale. The ACS assesses self-reported attentional control in terms of attentional focus, attentional switching and the capacity to quickly generate new thoughts (20 items, range 20-80; Cronbach's alpha in present study $=0.80$ ), by indicating agreement with items like 'I can quickly switch from one task to another' and 'I have a hard time concentrating when I'm excited about something'.

\section{Breath counting task}

The breath counting task was reproduced from Braboszcz and Delorme (2011). Participants were asked to keep their eyes closed and count their breath cycles (one inhalation and one exhalation) from 1 to 10 and then start from 1 again during two blocks of 20 minutes. They were instructed to press a button when they realized they had stopped counting, continued counting further than 10 , or when they had to reflect intensively on what the next count would be. Participants were instructed to refocus on breath-counting again after any button presses. In order to maintain procedural consistency with Braboszcz and Delorme (2011), a passive auditory oddball task was presented concurrently with the breath counting task and participants were instructed to ignore the auditory stimuli. We were not interested in studying oddball related EEG, but since it is possible that this particular detail of the procedure could influence mind wandering, we included it for the sake of close methodological replication. For the same reason, we also presented some debriefing questions at the end of each block (as done by Braboszcz and Delorme, 2011) that were not analyzed here.

\section{EEG recording and software}

EEG recordings were obtained continuously from 31 electrodes at 10/20 positions using Ag/AgCl electrodes of the ActiveTwo BioSemi system (BioSemi, The Netherlands). Electrodes placed on the left and right mastoids were used for offline re-referencing. Data were collected with a sampling rate of $1024 \mathrm{~Hz}$ with a gain of 16x at a bandwidth between DC-400 Hz. For processing purposes, data were down-sampled to $256 \mathrm{~Hz}$. 
Procedure

\section{General Procedure}

After informed consent had been obtained, participants completed the ACS and the STAI-t. This was followed by the measurement of resting-state EEG for ten minutes with eyes closed, and then the breath counting task was conducted while recording EEG.

Data Reduction

Button presses

For each subject, the EEG data were segmented into 16 second data epochs around their button presses. We considered that participants were mind wandering during the -8 to -2 second period preceding the button press, and that participants were concentrating on their breath during the 2 to 8 second period that followed the button press (as in Braboszcz \& Delorme, 2011). One participant pressed the button 111 times (more than 3 standard deviations above the mean number of button presses) and was therefore removed from further analysis. Twenty-seven subjects did not have enough clean (i.e., artefact free) data epochs to be considered for further analysis; specifically, these participants had below 11 button presses with EEG data of sufficient quality and were excluded.

\section{EEG pre-processing and FFT during baseline resting state}

EEG baseline data were re-referenced offline to the linked mastoids and automatically corrected for ocular artifacts (Gratton, Coles, \& Donchin, 1983) in segments of 4 seconds using Brain Vision Analyzer V2.04 (Brain Products GmbH, Germany). Baseline resting state EEG was then subjected to a Fast Fourier transformation (Hanning window length 10\%) to calculate power for the beta $(13-30 \mathrm{~Hz})$ and theta $(4-7 \mathrm{~Hz})$ band. Theta/beta ratio was calculated by dividing the theta by the beta power. All EEG baseline variables were non-normally distributed and therefore log-normalized with a $\log 10$ transformation. 
EEG pre-processing and FFT during the breath-counting task

For the EEG data during the breath counting task, offline re-referencing and ocular correction procedures were done as for the baseline EEG. Neuroscan 4.5 Edit software was then used to interpolate bad channels and extract single trial epochs for 8.25 second pre- to 8.25 second postbutton press. The remaining data quantification was completed within MATLAB (The Mathworks, Version 8.0.0.783, R2012b) using EEGLAB (Version 13.4; Delorme and Makeig, 2004). For each electrode and for each participant, 1 second intervals of sequential and non-overlapping data from 8 to 2 second before, and 2 to 8 second after each button press were individually selected, DC corrected, and a then 10\% Hanning window was applied. Discrete Fourier Transformation (DFT) was used to derive the frequency spectra at $1 \mathrm{~Hz}$ resolution, and a correction was applied for the use of the Hanning window. Wide band power data were then computed for the delta (1-3 Hz), theta (4-7 Hz), alpha (8-12 Hz) and beta $(13-30 \mathrm{~Hz})$ bands. Again, theta/beta ratio was calculated by dividing the theta power by the beta power. All EEG variables were non-normally distributed and therefore lognormalized with a $\log 10$ transformation.

Event-related spectral perturbations (ERSP) for EEG during the breath counting task.

To inspect differences in EEG pre-versus post-button press in more detail within the timefrequency domain, we computed Event Related Spectral Perturbations (ERSPs). For these analyses, we decomposed the EEG signal in brief overlapping segments using DFT. Each such ERSP used 257 sliding DFT windows with a size of 128 data points (500 ms). Data in each window were DC corrected, and a 10\% Hanning window was applied. Data were zero padded to 256 data points (1 second duration) and subjected to DFT. This gave us EEG power data at $1 \mathrm{~Hz}$ resolution, with a 62.5 ms time resolution. We assessed DC to $30 \mathrm{~Hz}$.

Each ERSP resulted in a three-dimensional matrix of EEG power at each frequency step and at each time point, containing all the information in the EEG throughout the trial. These ERSPs were obtained from 8 seconds before to 8 seconds after the button press for each trial and then averaged to obtain a mean ERSP for each subject. All ERSP derived EEG power values were non-normally distributed and therefore log-normalized with a log10 transformation. 
For the explorative topographical analyses, the following division in electrodes per region were made: Frontal; Fp1, Fp2, F3, Fz, F4, F7, F8, AFz, FCz, FC3, FC4, FT7, FT8; Central; C3, Cz, C4, CP3, CPz, CP4, T7, T8, TP7, TP8; Posterior; P3, Pz, P4, P7, P8, O1, Oz, O2; Left; Fp1, F7, F3, FT7, FC3, T7, C3, TP7, CP3, P7, P3, O1; Midline; AFz, Fz, FCz, Cz, CPz, Pz, Oz; Right; Fp2, F4, F8 FC4, FT8, C4, T8, CP4, TP8, P4, P8, O2.

\section{Results}

\section{Participants}

The 26 remaining participants had a mean age of 22.8 years ( $S D=2.6$, range: 19-28). Mean ACS score was $53.88(S D=5.44$, range 41-63), mean STAI-t score was $38.54(S D=6.32$, range 2950). The mean frontal TBR of the participants measured during the resting state (baseline) measurement was 1.22 ( $S D=0.49$, range 0.52-2.47 [non log-normalized]). All subjects had between 11 and 60 button presses $(M=23.76, S D=12.54)$.

\section{EEG activity pre- and post-button press average differences}

Theta was significantly higher pre- versus post-button press; $F(1,25)=13.60, p=0.001, \eta_{p}{ }^{2}=$ 0.35. Beta, on the other hand, was lower pre- compared to post-button press; $F(1,25)=18.58, p<$ $0.001, \eta_{p}{ }^{2}=0.43$. TBR was found to be significantly higher pre- compared to post-button press; $F(1,25)=28.05, p<0.001, \eta_{p}^{2}=0.53$. This confirms hypothesis I.

We exploratively tested pre- and post-differences for delta and alpha. Delta was significantly higher pre- versus post-button press; $F(1,25)=9.07, p=0.006, \eta_{p}{ }^{2}=0.27$. Alpha was significantly lower pre- versus post-button press; $F(1,25)=17.64, p<0.001, \eta_{p}^{2}=0.41$.

EEG theta/beta ratio baseline related to TBR change pre-versus post- and ACS.

Before testing whether differences in theta, beta or TBR pre- versus post-button press were affected by scores on the ACS, we checked whether there was a correlation between TBR change 
(pre- versus post-) and baseline TBR. No significant correlation was found between TBR change preversus post- and baseline TBR, $r=0.06 ; p=0.758$. We also tested whether there was a correlation between baseline TBR and ACS. When controlling for STAI-t, no significant correlation was found between ACS and baseline frontal TBR (partial $r=0.14, p=0.518$ ). This correlation was also absent without controlling for STAI-t; $r=0.16, p=0.423$. ACS did also not correlate significantly with frontal TBR as measured pre-button press ( $r=0.25, p=0.216)$, post-button press $(r=0.17, p=$ 0.404 ), or for the difference score of frontal pre-minus-post TBR; $r=0.17, p=0.540$. This was neither the case when controlling for STAI-t; ACS did not correlate with frontal TBR pre-button press (partial $r=0.25, p=0.220$ ), post-button press (partial $r=0.16, p=0.435$ ), or difference score of frontal pre-minus-post TBR; partial $r=0.15, p=0.483$. The lack of the TBR - ACS relation precludes the testing of hypotheses II, III and IV.

\section{Event Related Spectral Perturbations (ERSPs).}

To inspect the changes in the frequency bands of interest (theta, beta and TBR) pre-versus post-button press in more detail within the time-frequency domains, ERSP analyses were carried out. ERSP output included averages for all epochs of -8 to 8 seconds around the button press for all participants and all electrodes. As our hypotheses were based on previous findings with frontal TBR, we visualized ERSP data averaged over all participants of frontal electrode positions (average of F3, Fz and F4; Figure 1). This figure suggests that the power decreases post compared to pre-button press occurred not only in theta, but also in delta, while power increases were apparent not only in beta, but also alpha post-button press. Figure 1 also suggests that prior to the end of the post-button press epoch, theta power starts to increase again, and beta power starts to decrease.

\section{FIGURE 1 ABOUT HERE}

ERSP pre- and post-button press slopes and topography

To further focus on these time differences, mean narrow-band frontal ERSP data (across F3, 
Fz and F4) were summed to form the theta $(4-7 \mathrm{~Hz})$ and beta $(13-30 \mathrm{~Hz})$ frequency bands. These data were then averaged in 1 second non-overlapping sections to provide 6 averages from -8 to -2 seconds pre-button press, and 6 averages from 2 to 8 seconds post-button press. The same averages were calculated for theta/beta ratio by dividing the theta data by the corresponding data in beta. The slopes across the 6 pre- and 6 post averages for TBR are visualized in Figure 2.

A 2 (pre-post) x 6 (time-points) Multivariate analysis of variance (MANOVA) was conducted for Frontal TBR to explore the linear trend over the time points. A significant linear effect was found over pre- and post-, $F(1,25)=26.69, p<0.001, \eta_{p}{ }^{2}=0.52$. Frontal TBR did not have a significant linear slope trend over time pre-button press, $\left.F(5,25)=1.48, p=0.239, \eta_{p}^{2}=0.26\right)$, but (as can be seen in Figure 2) there was a significant linear slope trend over time post-button press, $F(5,25)=6.51, p=0.001, \eta_{p}{ }^{2}=0.61$, showing that TBR increased over time 2 to 8 seconds after the button press.

Furthermore, we exploratively evaluated topographical differences by conducting a 2 (prepost) x 3 (sagittal; frontal[F], central[C], posterior[P]) x 3 (lateral; left[L], midline[M], right[R]) MANOVA for TBR. TBR was dominant in the midline compared to the lateral regions ( $\mathrm{M}>\mathrm{L} / \mathrm{R}: \mathrm{F}=$ 66.96, $p<0.001, \eta_{p}{ }^{2}=0.73$ ), and in the frontal compared to the posterior regions (F $>\mathrm{P}: F=36.53, p$ $\left.<0.001, \eta_{p}^{2}=0.59\right)$. TBR also showed two-way interactions, with the midline dominance significantly larger in the frontal than posterior regions $\left(\mathrm{M}>\mathrm{L} / \mathrm{R} \times \mathrm{F}>\mathrm{P}: F=7.65, p=0.011, \eta_{p}{ }^{2}=\right.$ 0.23). The midline TBR dominance was also significantly larger in central compared to frontal/posterior regional mean ( $\mathrm{M}>\mathrm{L} / \mathrm{R}$ x C $\left.>\mathrm{F} / \mathrm{P}: F=15.61, p=0.001, \eta_{p}{ }^{2}=0.38\right)$. Pre- vs postbutton press interactions showed greater midline than lateral reductions $(\mathrm{M}>\mathrm{L} / \mathrm{R}$ x pre $>$ post: $F=$ 4.50, $\left.p=0.044, \eta_{p}{ }^{2}=0.15\right)$, particularly in posterior compared to frontal regions $(\mathrm{M}>\mathrm{L} / \mathrm{R} \mathrm{x} \mathrm{P}>\mathrm{F} \mathrm{x}$ pre $>$ post: $F=6.04, p=0.021, \eta_{p}^{2}=0.19$ ). Thus, the effect of MW on TBR was maximal in the posterior midline region.

ERSP data pre- and post-differences related to number of button presses.

As differences were found pre-versus post-button press, we explored whether these differences were related to the number of button presses that participants made. To analyse this, we 
first computed the average of the ERSP pre- (-8 to -2 seconds) and post-button press ( 2 to 8 seconds) and calculated the difference scores between these for frontal (average F3, Fz and F4) theta and beta band and the TBR. Correlational analysis showed no significant correlation between the number of button presses and the difference scores in theta $(r=0.07, p=0.741)$, beta $(r=-0.24, p=0.234)$, or TBR $(r=0.10, p=0.618)$. Thus, MW-related TBR change was independent of the number of button presses.

\section{FIGURE 2 ABOUT HERE}

\section{Discussion}

This study aimed to replicate and extend the design and results of Braboszcz and Delorme (2011) as pertaining to the MW related changes in EEG, to gain further insight into the role of frontal TBR during MW episodes. We found that frontal TBR was significantly higher during MW episodes compared to on-task time periods; this TBR - MW effect was strongest in the midline, particularly in posterior regions. When considering the EEG bands separately, theta power was higher and beta power was lower during MW episodes as opposed to on-task periods. Frontal baseline TBR did not correlate with ACS or the TBR-MW effect, resulting in an inability to test our hypothesis that previously observed relations between ACS and TBR might be explained by EEG changes during MW.

Our first hypothesis that frontal TBR would be higher during MW episodes was confirmed. TBR during MW episodes was stronger along the midline regions compared to the lateral regions and this effect was stronger in posterior compared to central and frontal regions. Previous crucial findings for TBR however, repeatedly assessed frontal TBR as measured frontally which was associated with prefrontally mediated cognitive and emotional processes (Putman et al., 2010, 2014; Angelidis et al., 2016; 2018; van Son et al., 2018a; Tortella-Feliu et al., 2014). For example, it predicted acute stressinduced changes in self-reported state attentional control in addition to its reported correlation with self-reported trait and state attentional control (Putman et al, 2014; Angelidis et al., 2016). Moreover, working memory training was found to decrease frontal TBR (Sari et al., 2015). Also, a theta-based brain stimulation procedure that has been shown to enhance working memory, decreased frontal and 
central TBR and increased flexible implicit rule learning in motivated decision making (Wischnewski, Zerr \& Schutter, 2016). Additionally, Schutter and van Honk (2005), used a reward-punishment reversal learning task to measure higher order cognitive integration of emotional information, and good performance on this same task correlated negatively with baseline frontal TBR; a similar result was also found in another more recent study (Schutte, Kenemans, Schutter, 2017). Additionally, several studies from our lab have provided evidence that resting-state frontal TBR predicted spatial attentional bias for threatening pictures, also interacting with individual differences in trait anxiety (Angelidis et al., 2018; van Son et al., 2018a). Relations between frontal TBR and attentional interference from high threat pictures were also altered by administration of caffeine, a catecholamine agonist that affects executive functioning in the PFC (van Son, Schalbroeck, Angelidis, van der Wee, van der Does \& Putman, 2018b).

Our data confirm and extend the findings by Braboszcz and Delorme (2011), and show that phasic changes in TBR are related to variation of mental state between uncontrolled MW and focused attention, or perhaps meta-cognitive vigilance. MW is thought to represent a state of reduced cognitive control (McVay \& Kane, 2009; Unsworth \& McMillan, 2014), reduced vigilant processing of external stimuli, and increased bottom-up, memory-driven self-referential thought (Mason et al., 2007). Changes in brain function that are associated with MW and these underlying cognitive processes include increased activation of the posterior cingulate cortex, medial PFC and parahippocampal regions - and decreased activation in (pre-frontal) cortical areas such as the dorso-lateral PFC and lateral inferior parietal regions (Hasenkamp et al., 2012; Karapanagiotidis, Bernhardt, Jefferies, \& Smallwood, 2017; Hopfinger, Buonocore, \& Mangun, 2000; Corbetta \& Schulman, 2002; Delaveaux, Arruda Sanchez, Steffen, Deschet, Jabourian, Perlbarg, \& Fossati, 2017). The current data then likely again support the conjecture that baseline TBR represents relative activation of top-down (prefrontal) cortical versus more bottom-up and subcortical processes, as first suggested by Schutter and van Honk (2005) and Knyazev (2007), and supported by our own work (Putman et al., 2010; 2014; Angelidis et al., 2018; van Son et al., 2018a), and that in several other labs (Schutter \& van Honk, 2004; Schutter \& van Honk, 2005; Sari et al., 2016; Tortella-Feliu et al., 2014; MorillasRomero et al., 2015; Keune et al., 2017; Clarke, Barry, McCarthy, \& Selikowitz, 2001). Additionally, 
the current confirmation that TBR may be used as a marker of MW-related changes in brain activity can likely be very useful for the study of MW (Smallwood \& Schooler, 2006) and inattention (Jap, Lal, Fischer, \& Bekiaris, 2009; Lorist, Bezdan, ten Caat, Span, Roerdink, \& Maurits, 2009).

The breath-counting MW method as used in this study and in Braboszcz and Delorme’s (2011) research (see also Hasenkamp et al., 2012, for a closely related method), has the potential limitation that it relies on introspection. Since the MW episodes that are examined are self-reported, their underlying brain activity might be different from other MW episodes that might have remained undetected, or earlier phases of the reported MW episodes. Also, it is perhaps not unlikely to assume that participants who were better able to realize that their mind wandered off the breath-counting, pressed the button more often, resulting in the results being driven by these participants. In other words, one could speculate that using the time periods before a button press might not capture episodes representative of all MW, but possibly predominantly MW episodes that are associated with more meta-attentional control or awareness. If this were so, one would expect that participants who are more aware of their MW episodes (and press the button more often than participants who are less aware of this) would show different EEG results. We tested if there was a correlation between the number of button presses and the TBR change, and this was not the case. The absence of this correlation is reassuring and likely indicates that the results are not confounded by meta-attentional introspective awareness. Also, if our results and the results from Braboszcz and Delorme (2011) would so partially reflect biased influence of MW episodes that are subsequently introspectively detected, one would expect that this should lead to a smaller pre- to post- button press effect. Thus, one could speculate that the results found using this method might underestimate the effect of mind wandering on TBR, if anything.

We used ERSP-derived one-second averages to further investigate slope changes over time in frontal TBR. The plotted slopes revealed that frontal TBR after a 'drop' that started just before the button press, increased again quite rapidly post-button press. This pattern of pre- versus post-button press raises an interesting speculation: is it really high TBR that we see during MW episodes, or perhaps rather low TBR shortly and briefly after the button press? Looking at the relatively fast rebound of frontal TBR, one explanation might be that individuals start to laps back into a new MW 
episode again relatively fast after the button press. We are however unsure how likely it is that they would start to mind wander again within eight seconds of becoming aware of their mind wandering. Another potentially interesting speculation concerning this seemingly quick rebound of frontal TBR is that the on-task focused periods might represent a short hypervigilant meta-awareness or metaattentional control (realising that one lost count and was mind wandering and subsequently increasing the use of executive resources for goal-directed monitoring of breath counting), which possibly contributed to the frontal TBR change pre- versus post-button press. This would be in line with literature on increased hypervigilance after error realization (e.g. Hollins, Harper, Gallagher, Owings, Lim, Miller et al., 2009; Weymar, Keil \& Hamm, 2013). This hypervigilance can be described as meta-cognition of one's attentional control and could possibly disappear relatively fast without having to engage back into a MW episode per se. It should be noted however, that such error realization is associated with short-lived increased theta activity (Hollins et al., 2009; Weymar et al., 2013), which seems at odds with our finding of decreased TBR (and theta) around the time of mind wandering realization. Future studies could take this speculation into account and compare MW periods with non-MW periods by using a design that does not rely on error related realizations, but utilizes experimenter-controlled probing questions about whether one is mind wandering.

All in all, the current results suggest frontal TBR to be related to changes in focused attention and possibly meta-attentional control or awareness. Beta is found to be involved in top- down executive functions like behavioural inhibition, inhibitory motoric processes, sequential encoding of processed items in working memory, retrieval from long-term memory and visual attention (Brown, 2007; Baker, 2007; Jenkinson \& Brown, 2011; Engel \& Fries, 2010; Marrufo, Vaquero, Cardoso, \& Gomez, 2001; Wróbel, 2000). Considering that beta activity has a strong coherence between frontal and parietal regions during top-down compared to bottom-up visual attention (Buschman \& Miller, 2007; 2009; Engel \& Fries, 2010) it was speculated that beta activity is to some extent related to the establishment of reciprocal control of bottom-up and top-down processes (Engel \& Fries, 2010). Theta activity on the other hand has been associated with subjective sleepiness (Strijkstra, Beersma, Drayer, Halbesma, \& Daan, 2003), decreased vigilance (e.g. Daniel, 1967; Belyavin, \& Wright, 1987), and was suggested to be generated in limbic structures involved in a brain network subserving 
more ‘bottom-up’ automatic attention as opposed to more cortically mediated executive control (Hermans et al., 2014; Seidenbecher, Laxmi, Stork, \& Pape, 2003). These lines of research fit with functional correlates of TBR and its role in mind wandering conceived as a state of reduced executive attentional control and automatic self-generated thought (Mason et al., 2007; McVay \& Kane, 2009; Unsworth \& McMillan, 2014; Smallwood, 2013; Christoff, Ream, Geddes, \& Gabrieli, 2003).

Exploratively, we additionally tested differences in the delta and alpha bands, and found that delta was significantly higher during MW episodes compared to on-task focus periods, while alpha was significantly higher during on-task focus periods compared to MW episodes. Changes in delta were similar to changes in theta, possibly because these bandwidths are adjacent and their functions have some possible overlap. Some studies have indeed described overlays in functionality for delta and theta in for example hippocampal - prefrontal coherent activity (Aleksanov, Vainstein \& Preobrashenskaya, 1986) and homeostatic and motivational processes (Knyazev, 2012). Putman et al. (2010) found similar correlations for theta/beta ratio and delta/beta ratio with fearful modulation of response inhibition in an emotional go/no-go task. Delta is classified, like theta, as a 'slow wave frequency', and their spectral proximity might therefore explain our similar current findings for delta and theta. As for alpha, a post-button press increase in power similar to beta was found. Alpha activity has been negatively related to vigilance, and positively related to inhibitory processes (Haegens, Luther, \& Jensen, 2012; see also Pfurtscheller, Stancak, \& Neuper, 1996; Klimesch, Sauseng, \& Hanslmayr, 2007, for reviews). Like beta, alpha is found to be involved in top-down processes, and more specifically, control over stored motoric information via inhibition of the retrieval of interfering information (e.g. Hummel, Andres, Altenmüller, Dichgans, \& Gerloff, 2002; Klimesch, 2012), and attentional control over sensory information (Wolfe \& Bell, 2004). Moreover, alpha activity was related to timing of neural activity to facilitate different behavioural states (Nicolelis \& Fanselow, 2002; Klimesch et al., 2007). As described above, beta is also related to top-down executive processes, which might explain why also alpha similarly varied as a function of mind wandering. The functions in which these bands are involved might have some overlap, explaining their similar increase during focused attention periods in the current results.

The expected correlations between baseline TBR, changes in TBR pre- versus post-button 
press and ACS were not found in the current study. A relation between baseline TBR and this difference in TBR during MW episodes and on-task periods would possibly affirm that higher TBR over the longer period of spontaneous TBR as measured during a typical resting state measurement is influenced by episodes of mind wandering, which could theoretically explain previously observed relations between such spontaneous TBR and attentional control and other cognitive executive processes (e.g. Putman et al., 2010; 2014; Angelidis et al., 2016). As the current sample showed no correlation between spontaneous TBR and ACS, this intriguing hypothesis could currently not be tested in the current study and should be revisited in future studies. The absence of a significant correlation between baseline frontal TBR and attentional control is contrary to several reports of this relation (Putman et al., 2010; 2014; Angelidis et al., 2016; van Son et al., 2018a; Keune et al., 2017; but see Morillas-Romero et al., 2015; Angelidis et al., 2018), which include negative correlations between TBR and subjectively as well as objectively measured attentional control. The current EEG measurements, both during baseline and the breath counting task, were recorded with only eyes closed to keep the procedure methodologically consistent with Braboszcz and Delorme (2011); this diverged from the alternating eyes-open-closed method that is typically used in previous studies of spontaneous TBR and indices of executive cognitive processes. Unpublished data from our lab, however, suggested no systematic differences between eyes-open or eyes-closed measurements in terms of frontal TBR in relation to other variables. It is therefore not clear if this difference in resting-state contributed to the absence of a frontal TBR-ACS relation in the current data. Note also that previous reports of TBR-ACS relations were based on larger samples than the current one. Still, TBR possibly relates more robustly to objective measures of attentional control, rather than to the self-report ACS as assessed here (as in Angelidis et al., 2018; van Son et al., 2018a; Morillas-Romero et al., 2015). Future studies might seek to investigate the relation between TBR and attentional control using both task-based and self-report measures of attentional control.

Since almost all previous TBR studies in healthy people (including studies examining attentional control) have looked at frontal TBR, this region was also selected as the a priori region of interest for the current study, as one of the aims was to directly establish relations between baseline frontal TBR, MW-related TBR and attentional control. The current topographical results, however, 
showed stronger midline dominance among the posterior region on TBR change pre- versus postbutton press. These results suggest that future studies investigating mind wandering should not restrict their analysis to frontal TBR, but should instead examine other regions as well.

Potential limitations of this study include that the implemented method causes a high (between-subjects) variance in the number of button presses. A substantial number of participants had to be excluded from analyses as they had too few analysable button presses or clean data epochs around the button press to reliably conduct analysis on (as in Braboszcz \& Delorme, 2011). However, we retained 26 participants which is more than twice the number of participants $(N=12)$ as assessed in Braboszcz and Delorme’s (2011) study, providing a robust replication of their proof of principle study. Also, the present study assessed only female participants which should be taken into account for the generalizability of our findings. Finally, although the results show a strong relation between scalp based EEG and mind wandering, and there is much evidence suggesting that TBR might reflect interactions between cortical and subcortical brain processes which could account for this finding, this interpretation remains based in indirect evidence. Future studies might attempt to revisit relations between TBR and mind wandering using for instance fMRI imaging to directly bridge this empirical gap.

In conclusion, this study confirms that increased frontal TBR is related to mind wandering and as such further supports the notion that low TBR reflects brain processes involved in executive control processes. The current findings contribute to a rudimentary understanding of the functional relation between frontal TBR and executive cognitive functions.

ACKNOWLEDGEMENTS: This study is supported by a grant from the Netherlands Organization for Scientific Research (NWO; \#452-12-003) to P.P. Collaboration between Leiden University and the University of Wollongong was supported by the Leiden University Fund (LUF; CWB \# 7518 SV) granted to D.v.S. NWO or LUF were not involved in any part of the current study. All authors were involved in the study design and approved the final manuscript. 


\section{References}

Aleksanov, S. N., Vainstein, I. I., \& Preobrashenskaya, L. A. (1986). Relationship between electrical potentials of the hippocampus, amygdala, and neocortex during instrumental conditioned reflexes. Neuroscience and behavioral physiology, 16(3), 199-207.

Angelidis, A., Hagenaars, M., van Son, D., van der Does, W., \& Putman, P. (2018). Do not look away! Spontaneous frontal EEG theta/beta ratio as a marker for cognitive control over attention to mild and high threat. Biological psychology, 135, 8-17.

Angelidis, A., van der Does, W., Schakel, L., \& Putman, P. (2016). Frontal EEG theta/beta ratio as an electrophysiological marker for attentional control and its test-retest reliability. Biological psychology, 121, 49-52.

Arns, M., Conners, C. K., \& Kraemer, H. C. (2013). A decade of EEG theta/beta ratio research in ADHD: a meta-analysis. Journal of attention disorders, 17(5), 374-383.

Baker, S. N. (2007). Oscillatory interactions between sensorimotor cortex and the periphery. Current opinion in neurobiology, 17(6), 649-655.

Barry, R. J., Clarke, A. R., \& Johnstone, S. J. (2003). A review of electrophysiology in attentiondeficit/hyperactivity disorder: I. Qualitative and quantitative electroencephalography. Clin Neurophysiol, 114(2), 171-183.

Belyavin, A., \& Wright, N. A. (1987). Changes in electrical activity of the brain with vigilance. Electroencephalography and clinical Neurophysiology, 66(2), 137-144.

Bishop, S. J. (2008). Neural mechanisms underlying selective attention to threat. Annals of the New 
York Academy of Sciences, 1129(1), 141-152.

Braboszcz, C., \& Delorme, A. (2011). Lost in thoughts: neural markers of low alertness during mind wandering. Neuroimage, 54(4), 3040-3047.

Brown, P. (2007). Abnormal oscillatory synchronisation in the motor system leads to impaired movement. Current opinion in neurobiology, 17(6), 656-664.

Buschman, T. J., \& Miller, E. K. (2007). Top-down versus bottom-up control of attention in the prefrontal and posterior parietal cortices. science, 315(5820), 1860-1862.

Buschman, T. J., \& Miller, E. K. (2009). Serial, covert shifts of attention during visual search are reflected by the frontal eye fields and correlated with population oscillations. Neuron, 63(3), 386-396.

Cavanagh, J. F., \& Shackman, A. J. (2015). Frontal midline theta reflects anxiety and cognitive control: meta-analytic evidence. Journal of Physiology-Paris, 109(1-3), 3-15.

Christoff, K., Ream, J. M., Geddes, L., \& Gabrieli, J. D. (2003). Evaluating self-generated information: anterior prefrontal contributions to human cognition. Behavioral neuroscience, 117(6), 1161.

Clarke, A. R., Barry, R. J., McCarthy, R., \& Selikowitz, M. (2001). EEG-defined subtypes of children with attention-deficit/hyperactivity disorder. Clinical Neurophysiology, 112(11), 2098-2105.

Corbetta, M., \& Shulman, G. L. (2002). Control of goal-directed and stimulus-driven attention in the brain. Nature reviews neuroscience, 3(3), 201.

Daniel, R. S. (1967). Alpha and theta EEG in vigilance. Perceptual and Motor Skills, 25(3), 697-703. 
Delaveau, P., Arruda Sanchez, T., Steffen, R., Deschet, K., Jabourian, M., Perlbarg, V., ... \& Fossati, P. (2017). Default mode and task-positive networks connectivity during the N-Back task in remitted depressed patients with or without emotional residual symptoms. Human brain mapping, 38(7), 34913501.

Derryberry, D., \& Reed, M. A. (2002). Anxiety-related attentional biases and their regulation by attentional control. Journal of Abnormal Psychology, 111(2), 225-236.

Engel, A. K., \& Fries, P. (2010). Beta-band oscillations—signalling the status quo?. Current opinion in neurobiology, 20(2), 156-165.

Gordon, N. (2000). REVIEW Cognitive functions and epileptic activity. Seizure-European Journal of Epilepsy, 9(3), 184-188.

Gregoriou, G. G., Rossi, A. F., Ungerleider, L. G., \& Desimone, R. (2014). Lesions of prefrontal cortex reduce attentional modulation of neuronal responses and synchrony in V4. Nature neuroscience, 17(7), 1003.

Gratton, G., Coles, M. G., \& Donchin, E. (1983). A new method for off-line removal of ocular artifact. Electroencephalography and Neurophysiology, 55, 468-484.

Haegens, S., Luther, L., \& Jensen, O. (2012). Somatosensory anticipatory alpha activity increases to suppress distracting input. Journal of cognitive neuroscience, 24(3), 677-685.

Hasenkamp, W., Wilson-Mendenhall, C. D., Duncan, E., \& Barsalou, L. W. (2012). Mind wandering and attention during focused meditation: a fine-grained temporal analysis of fluctuating cognitive states. Neuroimage, 59(1), 750-760. 
Hermans, E. J., Henckens, M. J., Joëls, M., \& Fernández, G. (2014). Dynamic adaptation of largescale brain networks in response to acute stressors. Trends in neurosciences, 37(6), 304-314.

Hirsch, C. R., \& Mathews, A. (2012). A cognitive model of pathological worry. Behaviour Research and Therapy, 50(10), 636-646.

Hollins, M., Harper, D., Gallagher, S., Owings, E. W., Lim, P. F., Miller, V., ... \& Maixner, W. (2009). Perceived intensity and unpleasantness of cutaneous and auditory stimuli: an evaluation of the generalized hypervigilance hypothesis. Pain, 141(3), 215-221.

Hopfinger, J. B., Buonocore, M. H., \& Mangun, G. R. (2000). The neural mechanisms of top-down attentional control. Nature neuroscience, 3(3), 284.

Hummel, F., Andres, F., Altenmüller, E., Dichgans, J., \& Gerloff, C. (2002). Inhibitory control of acquired motor programmes in the human brain. Brain, 125(2), 404-420.

Jap, B. T., Lal, S., Fischer, P., \& Bekiaris, E. (2009). Using EEG spectral components to assess algorithms for detecting fatigue. Expert Systems with Applications, 36(2), 2352-2359.

Jenkinson, N., \& Brown, P. (2011). New insights into the relationship between dopamine, beta oscillations and motor function. Trends in neurosciences, 34(12), 611-618.

Karapanagiotidis, T., Bernhardt, B. C., Jefferies, E., \& Smallwood, J. (2017). Tracking thoughts: Exploring the neural architecture of mental time travel during mind-wandering. Neuroimage, 147, 272-281.

Keune, P. M. , Hansen, A., Weber, E., Zapf, F., Habich, J., Muenssinger, J., Wolf, S., Schonenberg, 
M., Oschmann, P. (2017). Exploring neurophysiologic monitoring parameters and cognitive functioning in multiple sclerosis (MS): resting-state brain oscillatory activity and attention performance. Clinical neurophysiology

Klimesch, W. (2012). Alpha-band oscillations, attention, and controlled access to stored information. Trends in cognitive sciences, 16(12), 606-617.

Klimesch, W., Sauseng, P., \& Hanslmayr, S. (2007). EEG alpha oscillations: the inhibition-timing hypothesis. Brain research reviews, 53(1), 63-88.

Knyazev, G. G. (2007). Motivation, emotion, and their inhibitory control mirrored in brain oscillations. Neuroscience and Biobehavioral Reviews, 31, 377-395.

Knyazev, G. G. (2012). EEG delta oscillations as a correlate of basic homeostatic and motivational processes. Neuroscience \& Biobehavioral Reviews, 36(1), 677-695.

Lorist, M. M., Bezdan, E., ten Caat, M., Span, M. M., Roerdink, J. B., \& Maurits, N. M. (2009). The influence of mental fatigue and motivation on neural network dynamics; an EEG coherence study. Brain Research, 1270, 95-106.

Luu, P., Tucker, D. M., \& Makeig, S. (2004). Frontal midline theta and the error-related negativity: neurophysiological mechanisms of action regulation. Clinical Neurophysiology, 115(8), 1821-1835.

Marrufo, M. V., Vaquero, E., Cardoso, M. J., \& Gomez, C. M. (2001). Temporal evolution of $\alpha$ and $\beta$ bands during visual spatial attention. Cognitive Brain Research, 12(2), 315-320.

Mason, M. F., Norton, M. I., Van Horn, J. D., Wegner, D. M., Grafton, S. T., \& Macrae, C. N. (2007). Wandering minds: the default network and stimulus-independent thought. Science, 315(5810), 393- 
395.

Massar, S. A. A., Rossi, V., Schutter, D. J. L. G., \& Kenemans, J. L. (2012). Baseline EEG theta/beta ratio and punishment sensitivity as biomarkers for feedback-related negativity (FRN) and risk-taking. Clinical Neurophysiology, 123(10), 1958-1965.

McVay, J. C., \& Kane, M. J. (2009). Conducting the train of thought: working memory capacity, goal neglect, and mind wandering in an executive-control task. Journal of Experimental Psychology: Learning, Memory, and Cognition, 35(1), 196.

Mogg, K., \& Bradley, B. P. (1998). A cognitive-motivational analysis of anxiety. Behaviour research and therapy, 36(9), 809-848.

Mogg, K., \& Bradley, B. P. (2016). Anxiety and attention to threat: cognitive mechanisms and treatment with attention bias modification. Behaviour Research and Therapy, 87, 76-108.

Morillas-Romero, A., Tortella-Feliu, M., Bornas, X., \& Putman, P. (2015). Spontaneous EEG theta/beta ratio and delta-beta coupling in relation to attentional network functioning and selfreported attentional control. Cognitive, Affective, \& Behavioral Neuroscience, 15(3), 598-606.

Nicolelis, M. A., \& Fanselow, E. E. (2002). Thalamocortical optimization of tactile processing according to behavioral state. Nature neuroscience, 5(6), 517.

Nunez, P. L., \& Cutillo, B. A. (Eds.). (1995). Neocortical dynamics and human EEG rhythms. Oxford University Press, USA.

Ottaviani, C., Shahabi, L., Tarvainen, M., Cook, I., Abrams, M., \& Shapiro, D. (2015). Cognitive, 
behavioral, and autonomic correlates of mind wandering and perseverative cognition in major depression. Frontiers in neuroscience, 8, 433.

Pesaran, B., Nelson, M. J., \& Andersen, R. A. (2008). Free choice activates a decision circuit between frontal and parietal cortex. Nature, 453(7193), 406.

Pfurtscheller, G., Stancak Jr, A., \& Neuper, C. (1996). Event-related synchronization (ERS) in the alpha band — an electrophysiological correlate of cortical idling: a review. International journal of psychophysiology, 24(1-2), 39-46.

Putman, P., Van Peer, J., Maimari, I., \& Van der Werff (2010). EEG theta/beta ratio in relation to fear-modulated response-inhibition, attentional control, and affective traits. Biological Psychology, 83, 73-78.

Putman, P., Verkuil, B., Arias-Garcia, E., Pantazi, I., \& Van Schie, C. (2014). EEG theta/beta ratio as a potential biomarker for attentional control and resilience against deleterious effects of stress on attention. Cognitive, Affective, \& Behavioral Neuroscience, 14, 782-791.

Sari, B. A., Koster, E. H., Pourtois, G., \& Derakshan, N. (2016). Training working memory to improve attentional control in anxiety: A proof-of-principle study using behavioral and electrophysiological measures. Biological Psychology, 121, 203-212.

Schooler, J. W. (2002). Re-representing consciousness: Dissociations between experience and metaconsciousness. Trends in cognitive sciences, 6(8), 339-344.

Schutte, I., Kenemans, J. L., \& Schutter, D. J. (2017). Resting-state theta/beta EEG ratio is associated with reward-and punishment-related reversal learning. Cognitive, Affective, \& Behavioral Neuroscience, 17(4), 754-763. 
Schutter, D. J., \& Knyazev, G. G. (2012). Cross-frequency coupling of brain oscillations in studying motivation and emotion. Motivation and emotion, 36(1), 46-54.

Schutter, D. J. L. G., \& Van Honk, J. (2005). Electrophysiological ratio markers for the balance between reward and punishment. Cognitive Brain Research, 24(3), 685-690.

Schutter, D. J., \& Van Honk, J. (2004). Decoupling of midfrontal delta-beta oscillations after testosterone administration. International Journal of Psychophysiology, 53(1), 71-73.

Seidenbecher, T., Laxmi, T. R., Stork, O., \& Pape, H. C. (2003). Amygdalar and hippocampal theta rhythm synchronization during fear memory retrieval. Science, 301(5634), 846-850.

Shechner, T., \& Bar-Haim, Y. (2016). Threat monitoring and attention-bias modification in anxiety and stress-related disorders. Current Directions in Psychological Science, 25(6), 431-437.

Siegel, M., Donner, T. H., Oostenveld, R., Fries, P., \& Engel, A. K. (2008). Neuronal synchronization along the dorsal visual pathway reflects the focus of spatial attention. Neuron, 60(4), 709-719.

Smallwood, J., \& Schooler, J. W. (2006). The restless mind. Psychological bulletin, 132(6), 946.

Smallwood, J. (2013). Distinguishing how from why the mind wanders: a process-occurrence framework for self-generated mental activity. Psychological bulletin, 139(3), 519.

Strijkstra, A. M., Beersma, D. G., Drayer, B., Halbesma, N., \& Daan, S. (2003). Subjective sleepiness correlates negatively with global alpha $(8-12 \mathrm{~Hz})$ and positively with central frontal theta $(4-8 \mathrm{~Hz})$ frequencies in the human resting awake electroencephalogram. Neuroscience letters, 340(1), 17-20. 
Tortella-Feliu, M., Morillas-Romero, A., Balle, M., Llabrés, J., Bornas, X., \& Putman, P. (2014).

Spontaneous EEG activity and spontaneous emotion regulation. International Journal of Psychophysiology, 94(3), 365-372.

Unsworth, N., \& McMillan, B. D. (2014). Similarities and differences between mind-wandering and external distraction: A latent variable analysis of lapses of attention and their relation to cognitive abilities. Acta Psychologica, 150, 14-25.

van Son, D., Angelidis, A., Hagenaars, M.A., van der Does, W., Putman, P. (2018a) The role of individual differences in EEG theta/beta ratio, attentional control and trait anxiety in attentional bias to varying levels of threat. in submission.

van Son, D., Schalbroeck, R., Angelidis, A., van der Wee, N.J.A., van der Does, W., \& Putman, P. (2018b). Acute effects of caffeine on threat-selective attention: Moderation by anxiety and EEG theta/beta ratio. Biological psychology.

Weymar, M., Keil, A., \& Hamm, A. O. (2013). Timing the fearful brain: unspecific hypervigilance and spatial attention in early visual perception. Social cognitive and affective neuroscience, 9(5), 723729.

Wischnewski, M., Zerr, P., \& Schutter, D. J. (2016). Effects of Theta Transcranial Alternating Current Stimulation Over the Frontal Cortex on Reversal Learning. Brain stimulation, 9(5), 705-711.

Wolfe, C. D., \& Bell, M. A. (2004). Working memory and inhibitory control in early childhood: Contributions from physiology, temperament, and language. Developmental psychobiology, 44(1), 6883. 
Wróbel, A. (2000). Beta activity: a carrier for visual attention. Acta neurobiologiae experimentalis, 60(2), 247-260.

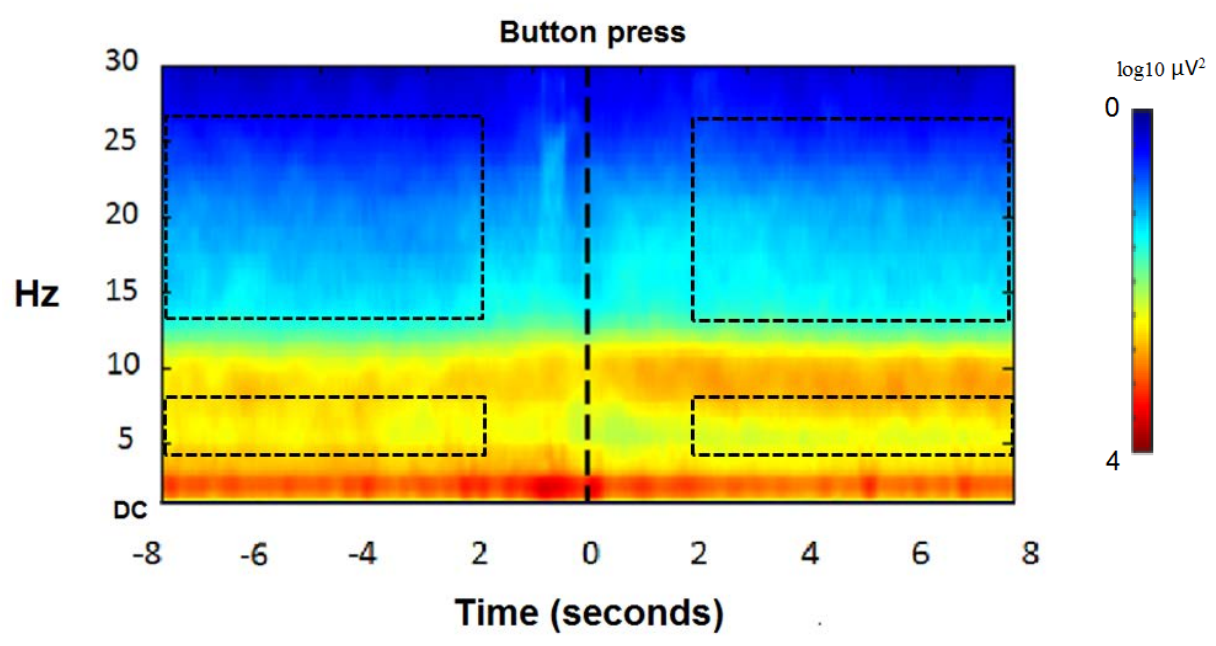

Figure 1, ERSP plot of the frontal average (across F3 Fz F4 sites) at $1 \mathrm{~Hz}$ frequency resolution, and $62.5 \mathrm{~ms}$ time resolution. Mind wandering was considered to have occurred in the -8 to -2 second period preceding the button press, and breath focus was considered to have occurred in the +2 to +8 second period following the button press. Rectangular frames highlight these data of interest. 


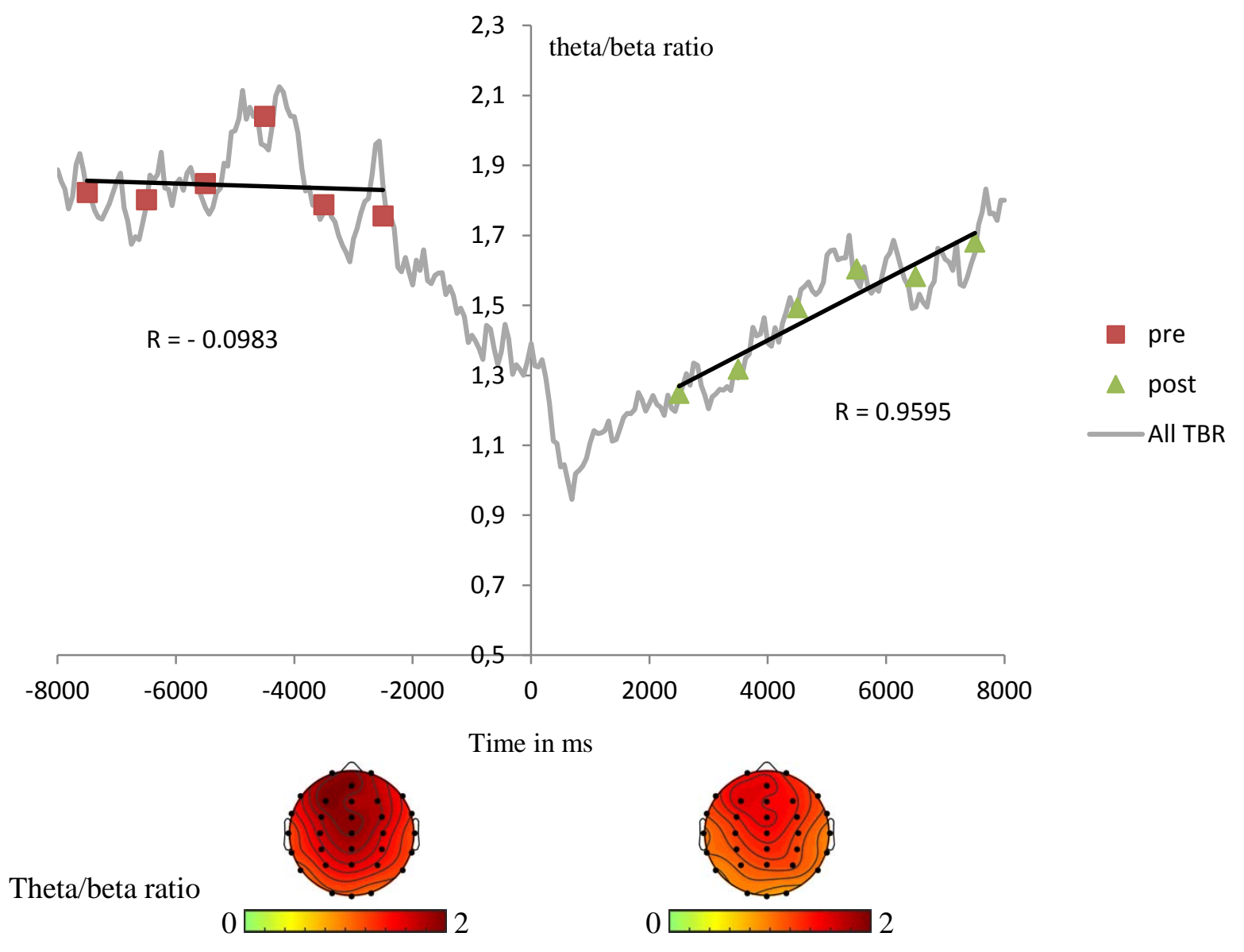

Figure 2. Plot of the ERSP-derived theta/beta ratio (TBR; non-logtransformed) data for Frontal electrode mean (F3, Fz and F4) showing slope trends plotted over six- 1 second averages pre- and post-button press.

Topographic map of power pre (left) and post (right) button press is shown for TBR averaged from -8 to -2 second before and 2 to 8 second after the button press. 